\title{
Integrating supplier selection, lot sizing and facility location decisions under a TBL approach: a case study
}

\author{
Mehmet Alegoz $^{1}$ (D) Haluk Yapicioglu ${ }^{1}$
}

Accepted: 28 January 2022 / Published online: 21 February 2022

(c) The Author(s), under exclusive licence to Springer-Verlag GmbH Germany, part of Springer Nature 2022

\begin{abstract}
Triple bottom line (TBL) is one of the well-known frameworks for measuring the sustainability of a system in which environmental and social aspects are also considered together with the economic aspect. In this study, we consider the integrated supplier selection, lot sizing and facility location decisions and propose a two-phase decision framework based on TBL approach. In the first phase of the study, we use a trapezoidal type-2 fuzzy AHP algorithm for the initial evaluation of the suppliers based on various economic, environmental and social criteria, whereas in the second phase of the study, we propose a two-stage stochastic programming model for the integrated supplier selection, lot sizing and facility location decisions under supply and demand uncertainties. We also present a real-life case study to test the proposed decision framework. Computational results, on one hand, show the applicability of the proposed decision framework on real-life cases and, on the other hand, bring significant managerial insights. Particularly, we observe that all three pillars (economic, environmental and social) of sustainability are in conflict with each other and thus being both environmentally and socially responsible is much more costlier than being solely environmentally or socially responsible. Moreover, we observe that ignoring the uncertainties in the system may yield to higher costs and impractical results.
\end{abstract}

Keywords Supplier selection $\cdot$ Lot sizing $\cdot$ Triple bottom line $\cdot$ Carbon cap policy $\cdot$ Fuzzy AHP $\cdot$ Stochastic optimization

\section{Introduction}

Having a good production planning and replenishment control through effective inventory management is crucial for all companies to be competitive in the market Lee et al. (2013). Companies need to work with numerous suppliers to continue their processes. In manufacturing industries, components may constitute up to $70 \%$ of the product costs and thus the purchasing department can play a significant role in cost reduction (Ustun and Demirtas (2008)). The problems of lot sizing and supplier selection focus on the determination of which suppliers to procure from, periods in which orders need to be placed and the lot sizes in order to meet demand in a timely manner Mazdeh et al. (2015). Due to its mathematical complexity, this problem is one of the most difficult problems in operations management (Rezaei et al. (2016)).

Mehmet Alegoz

mehmetalegoz@eskisehir.edu.tr

1 Department of Industrial Engineering, Eskisehir Technical University, 26555 Eskisehir, Turkey
This study proposes a framework for the integrated supplier selection, lot sizing and facility location decisions faced by the companies. Proposed decision framework particularly addresses the following questions.

i. Which raw materials should be procured from which suppliers?

ii. When should these raw materials be procured?

iii. What should be the lot size in each procurement?

iv. Where and how many manufacturing plants should be opened?

Note that the decisions regarding the above-mentioned questions affect each other, i.e. the location of the manufacturing plant affects the selected suppliers and vice versa. Hence, these decisions must be made in an integrated manner instead of tackling them independently.

Public awareness and regulations yield to a significant pressure on companies to decrease their carbon emissions. According to the State and Trends of Carbon Pricing (2020) report published by World Bank ${ }^{1}$, as of 2020, there are more

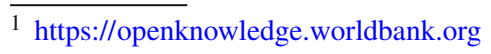


than sixty carbon pricing initiatives in place or scheduled for implementation in the near future. These initiatives force companies to consider the environmental aspect of their supply chains together with the economic aspect. In addition to environmental awareness, social awareness has also emerged as an important issue in manufacturing industries in recent years. For example, after the collapse of the Rana Plaza building in Dhaka, Bangladesh, a significant public pressure is observed and several of the world's largest apparel companies agreed to a landmark plan to help pay for fire safety and building improvements (Greenhouse and Yardley 2013). These discussions reveal that the companies must also consider the environmental and social aspects of the problem together with the economic aspect. In line with this fact, proposed integrated decision framework does not only focus on the economic aspect of the problem, but also considers the environmental and social aspects. By this way, it also guides the companies regarding how they can make their businesses more sustainable.

Proposed integrated decision framework includes two phases. In the first phase, an initial evaluation is made among suppliers based on various economic, environmental and social criteria by using a trapezoidal type- 2 fuzzy AHP algorithm and suppliers with the highest overall score are determined as candidate suppliers to work with. In the second phase, a two-stage stochastic programming model is proposed for the integrated supplier selection, lot sizing and facility location decisions under supply and demand uncertainties.

During the lifetime of a supply chain, various parameters are exposed to dramatic changes. Considering these parameters as deterministic is highly unrealistic and it could result in irrecoverable costs and inefficiencies (Hasani et al. 2012). In line with this fact, in this study uncertainty is taken into account in both two phases of the decision framework. In the first phase, uncertainty is handled by using a fuzzy MCDM approach instead of a classical MCDM approach, whereas in the second phase, demand and supply uncertainties are considered by using a stochastic programming approach.

The rest of the paper is organized as follows. After discussing the related literature in Section 2, we define the problem in Section 3 and present the proposed decision framework in Section 4. Section 5 is dedicated to a real-life case study and finally Section 6 is dedicated to conclusion and future work suggestions.

\section{Literature review}

In this section, we present the literature related to this study. To this end, we discuss the studies (i) focusing on the supplier evaluation and selection problem, (ii) focusing on the lot sizing problem and (iii) focusing on the effect of carbon emission and carbon regulations in supply chains. Finally, we discuss the motivation and contribution of this study based on the studies discussed.

Multi-criteria decision making (MCDM) approaches are among the most widely used approaches for supplier selection problem. For instance, Abdollahi et al. (2015) propose a framework that utilizes analytic network process (ANP) and data envelopment analysis (DEA) and Liu et al. (2019) propose a hybrid approach including quality function deployment (QFD) and VIKOR for the supplier evaluation and selection problem. There are also some studies that consider the uncertainty in system and hence use fuzzy MCDM approaches in supplier evaluation and selection process. For instance, (Karsak and Dursun 2015) develop a hybrid approach that utilizes QFD, along with fusion of fuzzy information and 2-tuple linguistic representation model, (Gupta et al. 2019) use a hybrid fuzzy MCDM approach based on AHP, TOPSIS, MABAC and WASPAS, and finally, (Tavassoli et al. 2020) use a stochastic fuzzy DEA for the supplier evaluation and selection problem. In addition to these studies, there are also some studies proposing novel fuzzy-logicbased approaches for solving problems under uncertainty (Chen et al. 2019, 2021b; De et al. 2022).

Another stream of research focuses on the lot sizing problem. For instance, (Basnet and Leung 2005) propose a model to determine what products to order in what quantities in which periods from which suppliers. Later, their model is solved by Cárdenas-Barrón et al. (2015) by using a new algorithm based on a reduce and optimize approach and a new valid inequality. (Razmi and Maghool 2010) propose a fuzzy bi-objective model considering capacity constraints, budget limitations and quantity discounts. Their model aims to minimize the total purchasing cost and maximize the total value of purchasing. In another study, (Rezaei and Davoodi 2011) develop a lot sizing model to investigate the effect of backlogging and observe that when backordering is allowed, better results in terms of cost, service level and total quality may be obtained. (Ghaniabadi and Mazinani 2017) consider incremental and all-units discount types and develop mixedinteger linear programming formulations. They also present a new recursive formulation providing optimal and/or near optimal solutions. (Kirschstein and Meisel 2019) consider the supplier selection and order allocation problem in process industry where procured raw material needs to be stored in heterogeneous storage facilities. The proposed model selects suppliers, decides which storage facilities to use, determine order quantities and transport flows under price discount policies of alternative suppliers.

Effects of carbon emission and carbon regulations on supply chains are also widely addressed in the existing literature. For example, (Kaur and Singh 2017) develop a mathematical model for order allocation problem for a multi-product, multi-period setting, to select from multiple 
Table 1 Existing literature

\begin{tabular}{|c|c|c|c|c|c|c|}
\hline & $\begin{array}{l}\text { Supplier } \\
\text { evaluation }\end{array}$ & $\begin{array}{l}\text { Supplier } \\
\text { lot } \\
\text { sizing }\end{array}$ & $\begin{array}{l}\text { Facility } \\
\text { location }\end{array}$ & $\begin{array}{l}\text { Supply and } \\
\text { demand } \\
\text { uncertainty }\end{array}$ & $\begin{array}{l}\text { Triple } \\
\text { bottom line } \\
\text { approach }\end{array}$ & $\begin{array}{l}\text { Carbon } \\
\text { cap-and } \\
\text {-trade }\end{array}$ \\
\hline Basnet and Leung (2005) & - & + & - & - & - & - \\
\hline Razmi and Maghool (2010) & + & + & - & - & - & - \\
\hline Rezaei and Davoodi (2011) & + & + & - & - & - & - \\
\hline Karsak and Dursun (2015) & + & - & - & - & - & - \\
\hline Azadnia et al. (2015) & + & + & - & - & + & - \\
\hline Cárdenas-Barrón et al. (2015) & - & + & - & - & - & - \\
\hline Ghaniabadi and Mazinani (2017) & - & + & - & - & - & - \\
\hline Kirschstein and Meisel (2019) & + & + & + & - & - & - \\
\hline Lamba et al. (2019) & + & + & - & - & - & + \\
\hline This study & + & + & + & + & + & + \\
\hline
\end{tabular}

suppliers and multiple logistics providers which also considers the carbon emissions in terms of sustainability. (Lamba et al. 2019) develop a mixed-integer nonlinear programming model aiming at minimizing the supply chain-wide costs and cost of carbon emissions under carbon cap, carbon cap-and-trade and carbon tax policies. (Bhattacharya and De 2021a) focus on emission sensitive supply chains and develop a model based on performance-based fuzzy game theory. (Bhattacharya and De 2021b) formulate a cost minimization EOQ model where the environmental pollution is incorporated. (De et al. 2021a) study a carbon emission sensitive partial backlogging EOQ model of COVID-19 materials where various issues such as the carbon tax policy and instantaneous deterioration have been incorporated. (De et al. 2021b) propose a new methodology for solving a pollution sensitive three-layer supply chain model of reworkable items. Choudhury et al. (2021) present an integrated single-vendor single-buyer production-inventory model that considers the environmental pollution and expiration date. Chen et al. (2021a) construct a new attribute system that considers environmental, social and economic factors for third-party reverse logistics provider selection. They state that in the evaluation process, emphasis must be given on social and environmental attributes in addition to traditional economic attributes in order to enhance the sustainable image of the enterprise.

Table 1 provides a comparison between this study and selected studies that are close to this study.

Based on the discussions above and Table 1, main motivation and contribution of this study can be summarized as follows.

- Although there are many studies considering supplier evaluation and selection problem (i.e. Karsak and Dursun (2015); Abdollahi et al. (2015); Liu et al. (2019); Gupta et al. (2019)) or lot sizing problem (i.e. Basnet and Leung (2005), Razmi and Maghool (2010); Ghaniabadi and Mazinani (2017), Lamba et al. (2019)), to the best of our knowledge, this study is the first study in the literature that propose a decision framework for the integrated supplier selection, lot sizing and facility location decisions under a TBL approach by considering various qualitative and quantitative criteria simultaneously.

- As it is shown in Table 1, most of the above-mentioned studies ignore the uncertainties in the system which may yield misleading and impractical results. In this study, uncertainty is considered in both phases of the proposed decision framework. Particularly, we use a trapezoidal type-2 fuzzy AHP algorithm in the first phase and stochastic programming in the second phase to handle the uncertainties. Consequences of ignoring uncertainties are also discussed.

- As Table 1 reveals, most of the above-mentioned studies focus only on the economic aspect of the problem. However, environmental and social aspects play a crucial role on the sustainability of a system and thus these aspects should also be considered. In line with this fact, environmental and social aspects of the supply chain are also considered together with the economic aspect in both first and second phases of the proposed decision framework. Effect of being environmentally and/or socially responsible is also discussed.

- This study presents a real-life case study based on a durable household goods manufacturer so as to show the applicability of the proposed decision framework to reallife cases.

\section{Problem definition}

In this study, we focus on a supply chain of a durable product such as household goods. The supply chain includes suppli- 
ers, manufacturing plants and retailers. The product requires different raw materials that need to be procured from different suppliers. Procured raw materials are shipped from suppliers to manufacturing plants and after the manufacturing operation, finished goods are shipped from manufacturing plants to retailers. Locations of the suppliers and retailers are fixed and known. However, the number and locations of the manufacturing plants need to be determined.

A fixed cost is incurred for each manufacturing plant opened. Moreover, each procurement from a supplier to a manufacturing plant yields to a fixed ordering cost that is independent from the amount of products procured and a variable procurement cost and shipment cost depending on the amount of products procured. Procured components, if not used in the same period, can be kept in the manufacturing plant at a unit holding cost per unit per period.

We consider a 12-month (1 year) planning horizon. In this planning horizon, demand of each retailer in each period is assumed to be uncertain. The product needs different raw materials to be manufactured and all the raw materials may not be supplied by all the suppliers. For instance, there may be two candidate suppliers for the procurement of a certain raw material but five candidate suppliers for the procurement of another raw material. Moreover, supply capacities of the suppliers for each raw material are also assumed to be uncertain over the planning horizon.

In this context, we propose a two-phase decision framework consisting of a trapezoidal type-2 fuzzy AHP algorithm in the first phase and a stochastic programming model in the second phase. First phase of the decision framework is dedicated to the initial evaluation of suppliers based on various qualitative and quantitative criteria and determination of the candidate suppliers to work with. In the second phase of the study, integrated supplier selection, lot sizing and facility location problem are solved by considering the candidate suppliers determined in the first phase. A real-life case study is also presented to test the efficiency and applicability of the proposed decision framework.

We introduce the proposed decision framework in the following section.

\section{Proposed decision framework}

In this section, we present the decision framework proposed for the integrated supplier selection, lot sizing and facility location decisions. The decision framework is illustrated in Figure 1. As the figure shows, the decision framework includes a trapezoidal type- 2 fuzzy AHP algorithm in the initial evaluation phase and a stochastic programming model in the integrated decision phase. These phases are explained in the following subsections.

\subsection{Initial evaluation phase}

Initial evaluation phase begins with the determination of the criteria. It is a well-known fact that various qualitative and quantitative criteria need to be considered in the supplier evaluation process. These criteria generally contradict with each other and thus the decision maker needs to find a trade-off between them. While determining the criteria, we consider two sources; (i) existing literature in the field and (ii) experts' opinions, i.e. opinions of the specialists and managers working in companies in the procurement or related departments. We observe that generally expert opinions and the literature are in agreement with each other. All in all, we determine seven economic, six environmental and four social criteria as presented in Table 2 .

Once the criteria are determined, the initial evaluation of the suppliers is made based on these criteria. For this purpose, a trapezoidal type- 2 fuzzy AHP algorithm is used and sup-

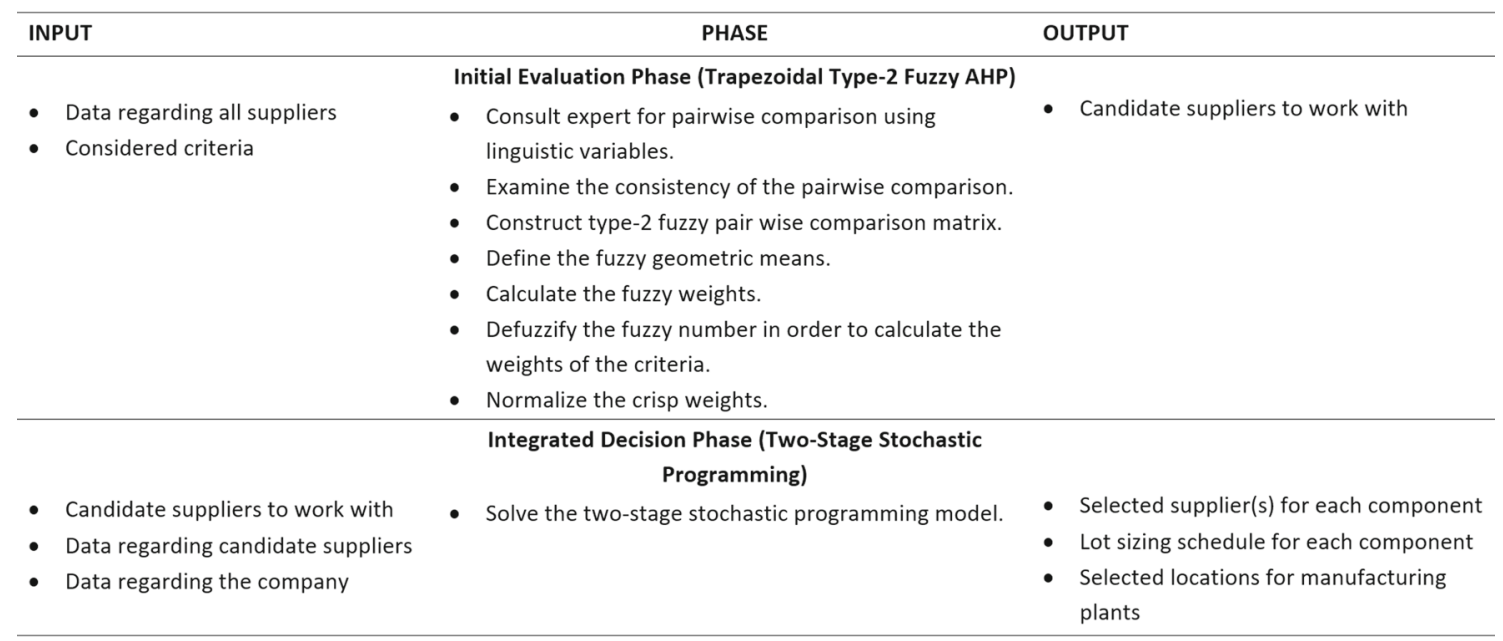

Fig. 1 Proposed decision framework 
Table 2 Criteria

\begin{tabular}{lll}
\hline Main criteria & Criteria & Reference \\
\hline Economic & Cost & Gupta et al. (2019) \\
Economic & Quality & Gupta et al. (2019) \\
Economic & Service level & Gupta et al. (2019) \\
Economic & Product and delivery reliability & Kaur et al. (2020) \\
Economic & After sale support & Kaur et al. (2020) \\
Economic & Financial health and reputation & Kaur et al. (2020) \\
Economic & R\&D and innovation & Abdollahi et al. (2015) \\
Environmental & Environmental responsibility & Kaur et al. (2020) \\
Environmental & Resource consumption & Büyüközkan and Berkol (2011) \\
Environmental & Fuel usage & Büyüközkan and Berkol (2011) \\
Environmental & Waste created & Büyüközkan and Berkol (2011) \\
Environmental & Amount of hazardous material & Liu et al. (2019) \\
Environmental & Greenhouse gas emission & Azadnia et al. (2015) \\
Social & Social responsibility & Liu et al. (2019) \\
Social & Ethical issues & Liu et al. (2019) \\
Social & Health and safety of employees & Liu et al. (2019) \\
Social & Training and education & Azadnia et al. (2015) \\
\hline
\end{tabular}

pliers that have a higher overall score than the predetermined threshold are considered as candidate suppliers to work with, while the remaining suppliers are eliminated.

In our analysis, we use type-2 fuzzy sets since they provide additional degrees of freedom to present the uncertainty and the fuzziness of the real world and thus more accurate and robust results can be obtained by using them (Buckley 1985; Chen and Lee 2010; Dereli and Altun 2013; Celik et al. 2014). Moreover, we use AHP since it allows to work with both qualitative and quantitative criteria, it provides a systematic way to check for the consistencies of judgements and it allows to construct a hierarchical structure.

Before introducing the steps of the used trapezoidal type-2 fuzzy AHP algorithm, it may be beneficial to give some brief definitions regarding trapezoidal type-2 fuzzy sets. For more detailed discussions, interested readers may refer to Buckley (1985), Chen and Lee (2010), Dereli and Altun (2013) and Celik et al. (2014). Following definitions and steps of the algorithm are obtained from these references.

Definition 1: A trapezoidal type-2 fuzzy set in the universe of discourse $X$ can be represented by a type- 2 membership function, shown as follows.

$$
\begin{array}{r}
\tilde{\tilde{A}}=\left\{\left((x, u), \mu_{\tilde{\tilde{A}}}(x, u)\right) \mid \forall x \in X, \forall u \in J_{X} \subseteq[0,1],\right. \\
\left.0 \leq \mu_{\tilde{\tilde{A}}}(x, u) \leq 1\right\}
\end{array}
$$

where $J_{X}$ denotes an interval in $[0,1]$. Moreover, the type-2 fuzzy set $\tilde{\tilde{A}}$ can also be represented as follows:

$$
\tilde{\tilde{A}}=\int_{x \in X} \int_{u \in J_{X}} \mu \tilde{\tilde{A}}(x, u) /(x, u)
$$

where $J_{X} \subseteq[0,1]$ and $\iint$ denotes union over all admissible $x$ and $u$.

Definition 2: Let $\tilde{\tilde{A}}$ be a type-2 fuzzy set in the universe of discourse $X$ represented by the type- 2 membership function $\mu_{\tilde{\tilde{A}}}$. If all $\mu_{\tilde{\tilde{A}}}(x, u)=1$, then $\tilde{\tilde{A}}$ is called as a trapezoidal interval type- 2 fuzzy set. A trapezoidal interval type-2 fuzzy set $\tilde{\tilde{A}}$ can be regarded as a special case of a type- 2 fuzzy set, represented as follows.

$\tilde{\tilde{A}}=\int_{x \in X} \int_{u \in J_{X}} 1 /(x, u)$ where $J_{X} \subseteq[0,1]$.

Definition 3: The upper membership function and the lower membership function of a trapezoidal interval type-2 fuzzy set are type-1 membership functions, respectively.

Based on these definitions, steps of the trapezoidal type-2 fuzzy AHP algorithm can be presented as follows. Please note that in the equations below, $\oplus$ and $\otimes$ refer to the summation operation between the trapezoidal interval type-2 fuzzy sets and multiplication operation between the trapezoidal interval type-2 fuzzy sets, respectively.

Step 1: Consult the expert for pairwise comparisons using linguistic variables.

Step 2: Examine the consistency of the pairwise comparisons. Step 3: Construct type-2 fuzzy pairwise comparison matrix among all the criteria in the hierarchical structure.

Step 4: Define the fuzzy geometric mean as follows

$\tilde{\tilde{r}}_{i}=\left(\tilde{\tilde{a}}_{i 1} \otimes \tilde{\tilde{a}}_{i 2} \otimes \ldots \otimes \tilde{\tilde{a}}_{i n}\right)^{1 / n}$. 
Step 5: Calculate the fuzzy weights by using the following equation.

$\tilde{\tilde{w}}_{i}=\tilde{\tilde{r}}_{i} \otimes\left(\tilde{\tilde{r}}_{1} \oplus \tilde{\tilde{r}}_{2} \oplus \ldots \oplus \tilde{\tilde{r}}_{n}\right)^{-1}$.

Step 6: Defuzzify the fuzzy number in order to calculate the weights of the criteria by using equation (6).

$$
\begin{aligned}
w_{i}^{\prime}= & \frac{1}{2}\left(\frac{1}{2} \sum_{i=1}^{4}\left(a_{i}^{L}+a_{i}^{L}\right)\right) \\
& \otimes \frac{1}{4}\left(\sum_{i=1}^{2}\left(H_{i}\left(A^{L}\right)+H_{i}\left(A^{U}\right)\right)\right) .
\end{aligned}
$$

Step 7: Normalize the crisp weights by using the following equation.

$w_{i}=\frac{w_{i}^{\prime}}{\sum_{i=1}^{n} w_{i}^{\prime}}$.

\subsection{Integrated decision phase}

In this phase of the study, we propose a two-stage stochastic programming model for the integrated supplier selection, lot sizing and facility location decisions. Sets, parameters and decision variables used in the model are presented in Table 3.

Based on this notation, the two-stage stochastic programming model can be presented as follows.

$$
\begin{aligned}
\min z= & \sum_{j} f_{j} \gamma_{j} \\
& +\sum_{s} p^{s}\left(\sum_{i} \sum_{j} \sum_{k} \sum_{t} \psi_{i j k t}^{s}\left(d_{i j} u+c_{i k}\right)\right. \\
& +\sum_{j} \sum_{l} \sum_{t} \delta_{j l t}^{s} e_{j l} u+\sum_{i} \sum_{t} \chi_{i t}^{s} o_{i} \\
& \left.+\sum_{j} \sum_{k} \sum_{t} \phi_{j k t}^{s} h_{k}\right) \\
& s . t \\
& \sum_{i} \sum_{j} \sum_{k} \sum_{t} \psi_{i j k t}^{s}\left(d_{i j} \hat{u}+\hat{c_{i k}}\right) \\
& +\sum_{j} \sum_{l} \sum_{t} \delta_{j l t}^{s} e_{j l} \hat{u} \\
& +\sum_{j} \sum_{k} \sum_{t} \phi_{j k t}^{s} \hat{h_{k} \leq} C_{e n v} \forall s \\
& \sum_{i} \sum_{j} \sum_{k} \sum_{t} \psi_{i j k t}^{s} m_{i} \\
& +\sum_{i} \sum_{j} \sum_{k} \sum_{t} \psi_{i j k t}^{s} n_{j} \geq C_{s o c} \quad \forall s
\end{aligned}
$$

$$
\begin{aligned}
& \phi_{j k 1}^{s}=\sum_{i} \psi_{i j k 1}^{s}-y_{k} \sum_{l} \delta_{j l 1}^{s} \forall j, k, s \\
& \phi_{j k t}^{s}=\phi_{j k(t-1)}^{s} \\
& \quad+\sum_{i} \psi_{i j k t}^{s}-y_{k} \sum_{l} \delta_{j l t}^{s} \forall j, k, s, \forall t \geq 2 \\
& \sum_{j} \psi_{i j k t}^{s} \leq a_{i k}^{s} \forall i, k, t, s \\
& \sum_{j} \delta_{j l t}^{s} \geq b_{l t}^{s} \forall l, t, s \\
& \sum_{j} \sum_{k} \psi_{i j k t}^{s} \leq M \chi_{i t}^{s} \quad \forall i, t, s \\
& \sum_{i} \sum_{k} \sum_{t} \sum_{s} \psi_{i j k t}^{s} \leq M \gamma_{j} \quad \forall j \\
& \psi_{i j k t}^{s}, \delta_{j l t}^{s}, \phi_{j k t}^{s} \geq 0 ; \chi_{i t}^{s}, \gamma_{j} \in\{0,1\} \quad \forall i, j, k, l, t, s .
\end{aligned}
$$

In the above model, equation (8) is the objective function minimizing the expected total cost. Equation (9) is the carbon cap constraint ensuring that the total emission will be less than or equal to the carbon cap in each scenario. Equation (10) is the social responsibility constraint ensuring that the social responsibility score of the company will be more than or equal to the predetermined level in each scenario. Equation (11) and (12) are the inventory balance constraints for manufacturing plants. Equation (13) makes sure that the supply capacity cannot be exceeded and equation (14) ensures that the demands must be satisfied . Equation (15) guarantees that an order must be made to make a shipment from a supplier and equation (16) ensures that the manufacturing plant must be opened to make a shipment from a supplier to that manufacturing plant. Finally, equation (17) is structural constraints that set the type and sign of the decision variables.

\section{A real-life case study}

In order to show the applicability of the proposed decision framework, in this section we present a real-life case study. We consider a household goods company in Turkey. We mainly focus on a specific product of the company, which we will refer to as Product A in the rest of the paper. Product A requires six different components. Particularly, 3 units of Component 1, 1 unit of Component 2, 1 unit of Component 3, 2 units of Component 4, 3 units of Component 5, 1 unit of Component 6 are needed to manufacture 1 unit of Product A.

There are 21 potential suppliers that can supply at least one of these six components required for Product $\mathrm{A}$. We denote them with the letters starting from A to U. In the initial evaluation phase, our aim is to determine the ten suppliers among these 21 suppliers that have the highest economic, environmental and social performance. 
Table 3 Notation

Sets

I:

$J:$

$K$ :

$L:$

$T:$

$S:$

Deterministic parameters

$d_{i j}$ :

$e_{j l}$ :

$f_{j}$ :

$o_{i}:$

$c_{i k}$ :

$y_{k}$ :

$h_{k}$ :

$u$ :

$\hat{c_{i k}}$ :

$\hat{h_{k}}$ :

$\hat{u}:$

$C_{\text {env }}$ :

$m_{i}$ :

$n_{j}:$

$C_{\text {soc }}$ :

$p^{s}$ :

$M$

Random parameters

$a_{i k}^{s}$ :

$b_{l t}^{s}$ :

Decision variables

$\psi_{i j k t}^{s}$ :

$\delta_{j l t}^{s}$ :

$\phi_{j k t}^{s}$ :

$\chi_{i t}^{s}$ :

$\gamma_{j}$ :
Set of suppliers indexed by $i$

Set of candidate locations for manufacturing plants indexed by $j$

Set of raw materials indexed by $k$

Set of retailers indexed by $l$

Set of periods indexed by $t$

Set of scenarios indexed by $s$

Distance between supplier $i$ and manufacturing plant in candidate location $j$

Distance between manufacturing plant in candidate location $j$ and retailer $l$

Fixed cost of opening manufacturing plant in candidate location $j$

Fixed cost of ordering from supplier $i$

Unit procurement cost of raw material $k$ supplied from supplier $i$

Amount of raw material $k$ needed for one unit of product

Unit holding cost of raw material $k$ per period

Unit shipment cost per unit per km

Unit emission dedicated to the manufacturing of raw material $k$ in supplier $i$

Unit emission of holding raw material $k$ per period

Unit shipment emission per unit per $\mathrm{km}$

Carbon cap

Social responsibility score of supplier $i$

Social responsibility score of manufacturing plant in candidate location $j$

Minimum social responsibility score that must be achieved

Probability that $s^{\text {th }}$ scenario occurs

A big number

Supply capacity of supplier $i$ for raw material $k$ under scenario $s$

Demand of retailer $l$ for product in period $t$ under scenario $s$

Amount of raw material $k$ shipped from supplier $i$ to plant $j$ in period $t$ under scenario $s$

Amount of product shipped from manufacturing plant $j$ to retailer $l$ in period $t$ under scenario $s$

Amount of raw material $k$ kept in manufacturing plant $j$ in period $t$ under scenario $s$

1 , if an order is made from supplier $i$ in period $t$ under scenario $s$, and 0 otherwise.

1 , if a manufacturing plant is opened in candidate location $j$, and 0 otherwise.

\subsection{Initial evaluation phase}

In this phase of study, we consider all 21 candidate suppliers and evaluate them based on the economic, environmental and social criteria determined in Table 2. For this purpose, we use the trapezoidal type-2 fuzzy AHP algorithm discussed in Section 4.1.

First, we collect the expert opinions and analyse the consistency of these opinions. Then, we create the type-2 fuzzy pairwise comparison matrix and obtain the fuzzy geometric means. For example, fuzzy geometric means for the main criteria are provided in Table 4.

Next, we calculate the fuzzy weights as presented in Table 5 for main criteria.
We continue in a similar manner and obtain the weights of the suppliers as in Figure 2.

Based on this initial evaluation, first-ten suppliers in Figure 2 are selected as the candidate suppliers for the procurement of the components of Product A, whereas the remaining eleven suppliers are eliminated. After selecting the ten candidate suppliers, we continue our analysis with the integrated decision phase.

\subsection{Integrated decision phase}

In this subsection, we focus on the integrated supplier selection, lot sizing and facility location decisions for Product A. As mentioned earlier, we consider both demand and supply 
Table 4 Fuzzy geometric means

Table 5 Fuzzy weights

Table 6 Scenario probabilities
C1:((1.26;1.44;1.44;1.59;1;1),(1.36;1.44;1.44;1.65;0.9;0.9))

C2:((1.00;1.00;1.00;1.00;1;1),(1.00;1.00;1.00;1.00;0.9;0.9))

C3:((0.63;0.69;0.69;0.79;1;1),(0.60;0.69;0.69;0.74;0.9;0.9))

C1:((0.37;0.46;0.46;0.55;1;1),(0.40;0.46;0.46;0.56;0.9;0.9))

C2:((0.30;0.32;0.32;0.35;1;1),(0.30;0.32;0.32;0.34;0.9;0.9))

C3:((0.19;0.22;0.22;0.27;1;1),(0.18;0.22;0.22;0.25;0.9;0.9))

\begin{tabular}{llllllllll}
\hline Scenario No. & 1 & 2 & 3 & 4 & 5 & 6 & 7 & 8 & 9 \\
\hline Demand & Low & Low & Low & Medium & Medium & Medium & High & High & High \\
S. Capacity & Low & Medium & High & Low & Medium & High & Low & Medium & High \\
Probability & 0.09 & 0.12 & 0.09 & 0.12 & 0.16 & 0.12 & 0.09 & 0.12 & 0.09 \\
\hline
\end{tabular}

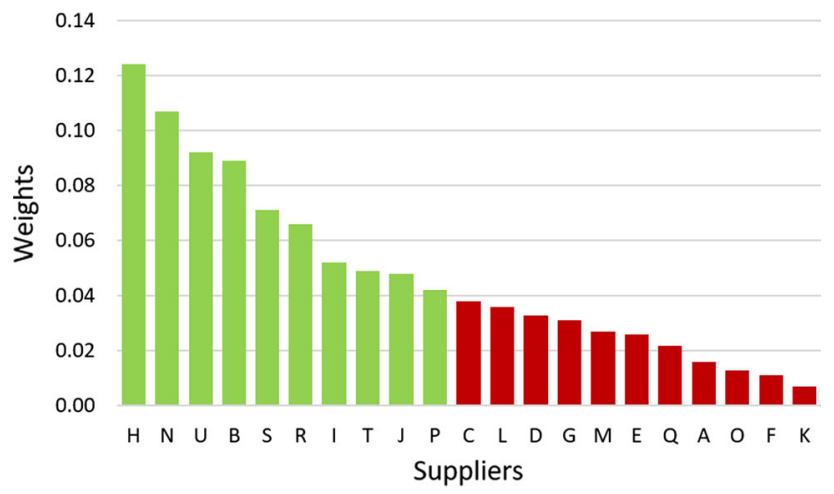

Fig. 2 Initial evaluation of suppliers

capacity uncertainties in our models. We consider three scenarios (low, medium, high) for each of these uncertainties which creates a total of nine scenarios. Probabilities of these scenarios are presented in Table 6.

In the previous phase of the study, we determined ten candidate suppliers to work with. Moreover, the company determined four candidate locations to open a manufacturing plant and there are seven regional retailers of the company in seven geographic regions of Turkey. Candidate suppliers, candidate locations for manufacturing plants and regional retailers are illustrated in the map in Figure 3. Distances between each of these facilities are obtained from the sources of Republic of Turkey General Directorate of Highways and Google Maps Service.

There are ten potential suppliers but note that all the suppliers do not manufacture all the components. For example, Supplier 1 does not manufacture Component 3 and Component 5. Moreover, some components are supplied by some suppliers in some scenarios but not supplied in some other scenarios. For example, Supplier 1 manufactures Component 1 in scenarios 4-9 but does not manufacture Component 1 in scenarios 1-3, i.e. Supplier 1 may or may not provide Component 1 . Similar to supply uncertainty, due to demand

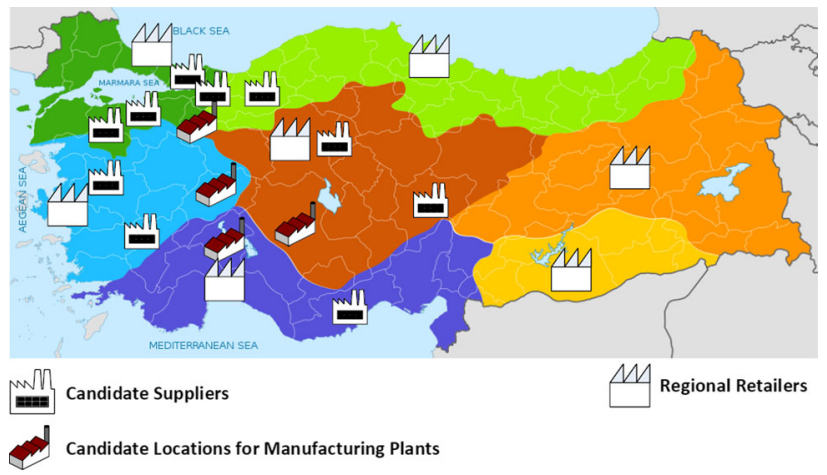

Fig. 3 Considered network of the company

uncertainty, demand of each retailer varies from a period to another and from a scenario to another.

Republic of Turkey Ministry of Industry and Technology periodically publish a report ${ }^{2}$ regarding the development levels of the cities and regions in Turkey. In this scientific report, cities and regions are evaluated by considering 52 sub-criteria in total under eight main criteria including demography, employment, education, health, competition, innovation, accessibility and quality of life. Based on this evaluation, a score is calculated for each city/region. We benefit from these scores while determining the social responsibility scores assuming that investing less developed regions yields to a higher social responsibility score for companies and vice versa. In this context, social responsibility scores of the suppliers and manufacturing plants are determined as in Table 7.

\footnotetext{
${ }^{2}$ https://www.sanayi.gov.tr
} 
Table 7 Social responsibility scores of the suppliers (S) and candidate manufacturing plants (MP)

\begin{tabular}{|c|c|c|c|c|c|c|c|c|c|c|c|c|c|}
\hline S1 & S2 & S3 & S4 & S5 & S6 & S7 & S8 & S9 & S10 & MP1 & MP2 & MP3 & MP4 \\
\hline 3.575 & 3.291 & 2.715 & 3.128 & 3.491 & 3.840 & 2.264 & 3.561 & 3.638 & 3.219 & 4.074 & 3.495 & 3.487 & 3.383 \\
\hline
\end{tabular}

Finally, emission factors for operations are obtained from publicly available sources such as Emission Factors for Greenhouse Gas Inventories Report. ${ }^{3}$

\subsection{Computational results}

In this subsection, we provide the computational results regarding the case study. First, we assume that the company does not consider the environmental and social aspects of the supply chain and makes the integrated supplier selection, lot sizing and facility location decisions by considering only the economic objective, i.e. minimizing the total cost. This base case serves us as a benchmark case while considering the environmental and social aspects.

For the base case instance, optimal cost, corresponding emission and corresponding social responsibility score are obtained as $\$ 10,320,220.16,486,380.00$ ton $-\mathrm{CO}_{2}$ and $2,995,300.00$, respectively. Moreover, the model also provides the optimal lot sizing schedule for each component. As an example, the lot sizing schedule for Component 1 is provided in Table 8 . Table 8 shows that the lot sizing schedule significantly changes from a scenario to another. For instance, an order is made in Period 4 in first-three scenarios but there is no order in Period 4 in remaining six scenarios.

Next, in order to test the effect of uncertainty, we first solve the model with deterministic parameters, i.e. by using the expected values of the uncertain parameters. Then, we fix the scenario-independent decisions (i.e. location decisions) obtained in deterministic model and solve the two-stage stochastic optimization model with those fixed decisions. By this way, we investigate the effect of using deterministic model decisions in an uncertain environment, i.e. in real-life. Our analysis reveals that ignoring the uncertainties may cause an increase in overall costs, about $8 \%$ in our instance. Hence, we can infer that considering the uncertainties in the system is crucial and ignoring this issue may yield to misleading results and higher costs in real-life applications.

Secondly, we assume that the company works under a carbon cap policy and investigate how the model decisions and performance measures change under this policy. Computational results are presented in Figure 4.

Various inferences can be made based on Figure 4. First, we observe that considering the environmental aspect yields to a substantial change in both lot sizing and facility location decisions of the company. Secondly, Figure 4 shows

\footnotetext{
${ }^{3}$ https://www.epa.gov
}

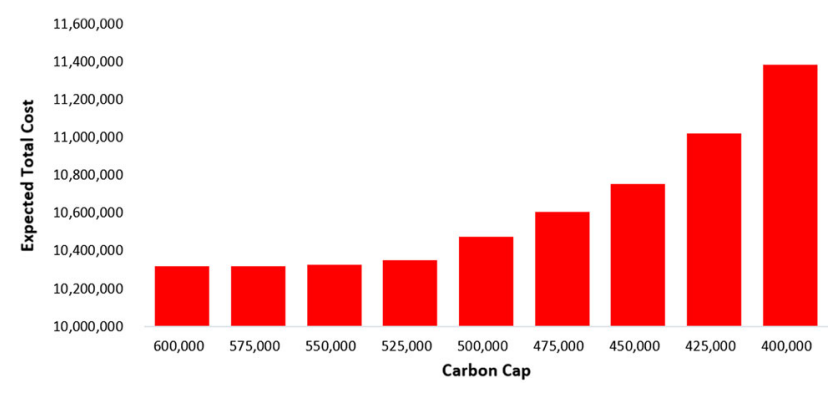

Fig. 4 Expected costs for different carbon caps

that decreasing the carbon cap brings a lower additional cost at lower carbon caps but higher additional costs at higher carbon caps. Hence, it is possible to claim that increasing the environmental responsibility level may be easier and less costly up to a certain value but after that value being more environmentally responsible brings a significant additional cost.

Thirdly, we assume that the company considers the economic and social aspects of the supply chain simultaneously. For this purpose, we test the model for different social responsibility scores and present the computational results in Figure 5.

Figure 5 shows that similar to being environmentally responsible, being socially responsible also brings a significant additional cost to companies and has a substantial effect on model decisions. This additional cost is less under lower social responsibility levels, but more under higher responsibility levels. Hence, we can conclude that similar to environmental responsibility level, social responsibility level should also be determined carefully by the companies such that a trade-off between economic and social performance is obtained.

Finally, we focus on both environmental and social performance and try to investigate the economic effect of being both environmentally and socially responsible. For this purpose,

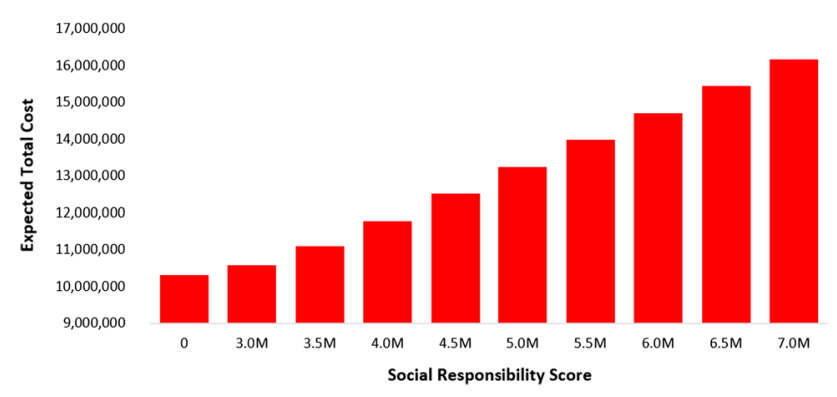

Fig. 5 Expected costs for different social responsibility scores 
Table 8 Lot sizing schedule for component 1

\begin{tabular}{lllllllllllll}
\hline & 1 & 2 & 3 & 4 & 5 & 6 & 7 & 8 & 9 & 10 & 11 & 12 \\
\hline 1 & 24,336 & - & - & 16,896 & - & 24,120 & - & - & 17,616 & - & 18,192 & - \\
2 & 24,336 & - & - & 16,896 & - & 24,120 & - & - & 17,616 & - & 18,192 & - \\
3 & 24,336 & - & - & 16,896 & - & 24,120 & - & - & 17,616 & - & 18,192 & - \\
4 & 19,860 & - & 20,730 & - & 22,080 & - & 19,020 & - & 22,020 & - & 22,740 & - \\
5 & 19,860 & - & 20,730 & - & 22,080 & - & 19,020 & - & 22,020 & - & 22,740 & - \\
6 & 19,860 & - & 20,730 & - & 22,080 & - & 19,020 & - & 22,020 & - & 22,740 & - \\
7 & 23,832 & - & 24,876 & - & 26,496 & - & 22,824 & - & 26,424 & - & 27,288 & - \\
8 & 23,832 & - & 24,876 & - & 26,496 & - & 22,824 & - & 26,424 & - & 27,288 & - \\
9 & 23,832 & - & 24,876 & - & 26,496 & - & 22,824 & - & 26,424 & - & 27,288 & - \\
\hline
\end{tabular}

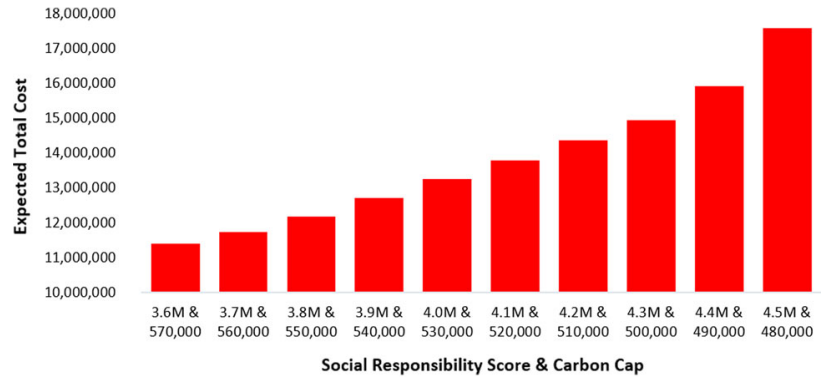

Fig. 6 Expected costs for different carbon caps and social responsibility scores

we test the models for different carbon cap and social responsibility score values. Computational results are presented in Figure 6. As Figure 6 shows, we simultaneously decrease the carbon cap and increase the social responsibility score in each instance. It should be noted that after a certain value, a further increase in social responsibility level and a further decrease in carbon cap yields to an infeasible solution.

Our analysis reveals that increasing the environmental responsibility level does not necessarily yield to an increase in social responsibility level. Contrarily, environmental and social aspects also have a conflict with each other. Thus, increasing both the environmental and social responsibility levels is much more costly compared to increasing either the environmental or the social responsibility level. Since all three pillars of the sustainability are in conflict with each other, a trade-off between them can only be found by joint consideration of them all.

\section{Conclusion and future work suggestions}

In this study, we propose a two-phase decision framework for the integrated supplier selection lot sizing and facility location decisions. In the first phase of the study, we use a trapezoidal type-2 fuzzy AHP algorithm for the initial evaluation of the suppliers based on various economic, environmental and social criteria, whereas in the second phase, we propose a two-stage stochastic programming model to make the integrated supplier selection, lot sizing and facility location decisions under demand and supply capacity uncertainties. We also present a real-life case study to test the proposed decision framework. In addition to demonstrating the applicability of the proposed decision framework in reallife cases, presented real-life case study also brings various managerial insights for us.

First, our analysis reveals that consideration of the uncertainties in the supply chains plays a crucial role on the success and applicability of the proposed decision framework in real-life cases. Ignoring uncertainties may yield to higher cost/emission values and may result in lot sizing schedules that are infeasible in real-life. Hence, companies should always consider the uncertainties in the system while making these decisions. Second, it is observed that increasing the environmental or social responsibility level brings a lower additional cost in lower levels but a higher additional cost in higher levels and thus the companies should carefully determine these levels such that a trade-off between cost and environmental/social responsibility is agreed upon. Third, our results reveal that increasing the environmental responsibility level does not necessarily yield to an increase in social responsibility level. Contrarily, environmental and social aspects also are in conflict with each other. Hence, being both environmentally and socially responsible is much more costly compared to being only environmentally or only socially responsible. Moreover, joint consideration of the economic, environmental and social aspects is essential to find a trade-off among them.

Following future work suggestions may be beneficial for interested readers. First, in this study, we consider only demand and supply uncertainties. Various other uncertainties such as the uncertainties in price, quality and lead time may be considered and the effects of these uncertainties to the decisions can be studied. Secondly, uncertainty can be handled by different approaches than stochastic programming. For instance, fuzzy programming may be a suitable option for this purpose. At this point, a study comparing the results obtained with different methods, i.e. stochastic programming and fuzzy programming may also bring significant insights 
regarding the superiority of one method to another. Third, different manufacturing approaches such as Just-in-Time can be considered in this decision framework and effects of these approaches on the economic, environmental and social performance measures can be studied. Finally, investigating the effects of well-known issues such as incremental quantity discount, all-units quantity discount, lot size dependent lead time and disruption risks may bring significant managerial insights regarding the procurement decisions in real-life applications.

Funding This study is supported by Eskisehir Technical University Scientific Research Projects Commission under the Grant Numbers 20ADP203 and 20ADP240.

Availability of data and materials (data transparency): Not applicable.

Code Availability Not applicable.

\section{Declarations}

Conflicts of interest Not applicable.

\section{References}

Abdollahi M, Arvan M, Razmi J (2015) An integrated approach for supplier portfolio selection: Lean or agile? Expert Syst Appl 42(1):679-690

Azadnia AH, Saman MZM, Wong KY (2015) Sustainable supplier selection and order lot-sizing: an integrated multi-objective decision-making process. Int J Prod Res 53(2):383-408

Basnet C, Leung JM (2005) Inventory lot-sizing with supplier selection. Comput Op Res 32(1):1-14

Bhattacharya K, De SK (2021a) A robust two layer green supply chain modelling under performance based fuzzy game theoretic approach. Comput Indus Eng 152:107005

Bhattacharya K, De SK (2021b) Solution of a pollution sensitive EOQ model under fuzzy lock leadership game approach. Granular Computing, 1-17

Buckley JJ (1985) Fuzzy hierarchical analysis. Fuzzy Sets Syst 17(3):233-247

Büyüközkan G, Berkol Ç (2011) Designing a sustainable supply chain using an integrated analytic network process and goal programming approach in quality function deployment. Expert Syst Appl 38(11):13731-13748

Cárdenas-Barrón LE, González-Velarde JL, Treviño-Garza G (2015) A new approach to solve the multi-product multi-period inventory lot sizing with supplier selection problem. Comput Op Res 64:225232

Celik E, Gumus AT, Alegoz M (2014) A trapezoidal type-2 fuzzy MCDM method to identify and evaluate critical success factors for humanitarian relief logistics management. J Intell Fuzzy Syst 27(6):2847-2855

Chen SM, Lee LW (2010) Fuzzy multiple attributes group decisionmaking based on the interval type-2 TOPSIS method. Expert Syst Appl 37(4):2790-2798

Chen ZS, Yang Y, Wang XJ, Chin KS, Tsui KL (2019) Fostering linguistic decision-making under uncertainty: a proportional interval type-2 hesitant fuzzy TOPSIS approach based on Hamacher aggregation operators and andness optimization models. Info Sci 500:229-258

Chen ZS, Zhang X, Govindan K, Wang XJ, Chin KS (2021a) Third-party reverse logistics provider selection: a computational semantic analysis-based multi-perspective multi-attribute decision-making approach. Expert Syst Appl 166:114051

Chen ZS, Zhang X, Rodríguez RM, Pedrycz W, Martínez L (2021b) Expertise-based bid evaluation for construction-contractor selection with generalized comparative linguistic ELECTRE III. Auto Const 125:103578

Choudhury M, De SK, Mahata GC (2021) Pollution-sensitive integrated production-inventory management for deteriorating items with quality loss and quantity loss with expiration date. International Journal of Systems Science: Operations \& Logistics, 1-23

De SK, Mahata GC, Maity S (2021a) Carbon emission sensitive deteriorating inventory model with trade credit under volumetric fuzzy system. Int J Intell Syst 36(12):7563-7590

De SK, Bhattacharya K, Roy B (2021b) Solution of a pollution sensitive supply chain model under fuzzy approximate reasoning. Int J Intell Syst 36(10):5530-5572

De SK, Roy B, Bhattacharya K (2022) Solving an EPQ model with doubt fuzzy set: a robust intelligent decision-making approach. Knowl-Based Syst 235:107666

Dereli T, Altun K (2013) Technology evaluation through the use of interval type-2 fuzzy sets and systems. Comput Indus Eng 65(4):624-633

Ghaniabadi M, Mazinani A (2017) Dynamic lot sizing with multiple suppliers, backlogging and quantity discounts. Comput Indus Eng 110:67-74

Greenhouse S, Yardley J (2013) Global Retailers Join Safety Plan for Bangladesh, New York Times, 13.05.2013, https://www.nytimes. com

Gupta S, Soni U, Kumar G (2019) Green supplier selection using multicriterion decision making under fuzzy environment: a case study in automotive industry. Comput Indus Eng 136:663-680

Hasani A, Zegordi SH, Nikbakhsh E (2012) Robust closed-loop supply chain network design for perishable goods in agile manufacturing under uncertainty. Int J Prod Res 50(16):4649-4669

Karsak EE, Dursun M (2015) An integrated fuzzy MCDM approach for supplier evaluation and selection. Comput Indus Eng 82:82-93

Kaur H, Singh SP (2017) Modeling low carbon procurement and logistics in supply chain: a key towards sustainable production. Sustain Prod Consum 11:5-17

Kaur H, Singh SP, Garza-Reyes JA, Mishra N (2020) Sustainable stochastic production and procurement problem for resilient supply chain. Comput Indus Eng 139:105560

Kirschstein T, Meisel F (2019) A multi-period multi-commodity lotsizing problem with supplier selection, storage selection and discounts for the process industry. Euro J Op Res 279(2):393-406

Lamba K, Singh SP, Mishra N (2019) Integrated decisions for supplier selection and lot-sizing considering different carbon emission regulations in Big Data environment. Comput Indus Eng 128:10521062

Lee AH, Kang HY, Lai CM, Hong WY (2013) An integrated model for lot sizing with supplier selection and quantity discounts. Appl Math Model 37(7):4733-4746

Liu A, Xiao Y, Lu H, Tsai SB, Song W (2019) A fuzzy three-stage multiattribute decision-making approach based on customer needs for sustainable supplier selection. J Clean Prod 239:118043

Mazdeh MM, Emadikhiav M, Parsa I (2015) A heuristic to solve the dynamic lot sizing problem with supplier selection and quantity discounts. Comput Indus Eng 85:33-43

Razmi J, Maghool E (2010) Multi-item supplier selection and lotsizing planning under multiple price discounts using augmented $\epsilon$-constraint and Tchebycheff method. Int J Adv Manuf Technol 49(1):379-392 
Rezaei J, Davoodi M (2011) Multi-objective models for lot-sizing with supplier selection. Int J Prod Econo 130(1):77-86

Rezaei J, Davoodi M, Tavasszy L, Davarynejad M (2016) A multiobjective model for lot-sizing with supplier selection for an assembly system. Int J Log Res Appl 19(2):125-142

Tavassoli M, Saen RF, Zanjirani DM (2020) Assessing sustainability of suppliers: a novel stochastic - fuzzy DEA model. Sustain Prod Consum 21:78-91
Ustun O, Demirtas EA (2008) Multi-period lot-sizing with supplier selection using achievement scalarizing functions. Comput Indus Eng 54(4):918-931

Publisher's Note Springer Nature remains neutral with regard to jurisdictional claims in published maps and institutional affiliations. 